\title{
Large-Scale Structure in Mixed Dark Matter Models with a Non-thermal Volatile Component
}

\author{
Elena PIERPAOLI ${ }^{1,2,3}$, Peter COLES $^{2}$, \\ Silvio BONOMETTO ${ }^{3,4}$ \& Stefano BORGANI ${ }^{1,4}$ \\ 1 SISSA - International School for Advanced Studies, \\ via Beirut 2-4, I-34013 Trieste, Italy \\ 2 Astronomy Unit, School of Mathematical Sciences, \\ Queen Mary $\& 5$ Westfield College, Mile End Road, \\ London E1 4NS, United Kingdom \\ ${ }^{3}$ INFN - Sezione di Milano, \\ Dipartimento di Fisica dell' Università di Milano, \\ via Celoria 16, I-20133 Milano, Italy \\ 4 INFN, Sezione di Perugia, \\ c/o Dipartimento di Fisica dell' Università, \\ via A. Pascoli, I-06100 Perugia, Italy
}




\begin{abstract}
We investigate the properties of large-scale structure predicted in a class of mixed dark matter models in which the volatile component (made of particles with high rms velocity) derives from the decay of a heavier particle. Such models based on cold+volatile dark matter (CVDM) differ from the standard mixture of CDM and massive neutrinos, usually known as CHDM, in that they involve a component which has a non-thermal phase space distribution function. As a consequence, and differently from CHDM models, the value of the redshift at which volatile particles become non relativistic, $z_{n r}$, can be varied almost independently of the volatile fraction, $\Omega_{X}$. We compute transfer functions for a selection of such models, having $0.1 \leq \Omega_{X} \leq 0.5$ and different values of $z_{n r}$. Using linear theory and assuming a scalefree primordial spectrum, we compare such models with observational constraints on largescale galaxy clustering and bulk flows, as well as on the abundance of galaxy clusters and high-redshift damped Ly $\alpha$ systems. We find that these constraints enable us to discriminate between different $\Omega_{X}$ and $z_{n r}$; within the range of the models inspected, those which can be most easily accommodated by the data correspond to the parameter choice $\Omega_{X} \simeq 0.2$ and $z_{n r} \simeq 2 \times 10^{4} \Omega_{X}$.
\end{abstract}

Subject headings: Cosmology: large-scale structure of the universe - galaxies: clusters: general 


\section{Introduction}

The outstanding problem of modern cosmology is to understand the formation of galaxies, clusters of galaxies and large-scale structure in the expanding universe. A scenario in which these structures form by the growth by gravitational instability of small-amplitude primordial density fluctuations in a universe dominated by weakly interacting non-baryonic dark matter has been the standard framework within this problem has been discussed for many years. For most purposes the crucial theoretical quantity in the gravitational instability picture is the primordial fluctuation spectrum, $P(k)$. In most theories, density perturbations originate as a quantum phenomenon in the very early universe with a power-law spectrum of the form $P(k) \propto k^{n} ; n$ very close to unity is favoured by many versions of the inflationary universe picture, as well as by more general considerations. With the discovery of temperature anisotropies in the Cosmic Microwave Background Radiation (CMBR) by the COBE satellite (Smoot et al. 1992), it is possible to fix the amplitude of this spectrum in a relatively unambiguous way on very large scales, of order $1000 \mathrm{Mpc}$. On smaller scales, the shape of the primordial spectrum is expected to evolve from its initial power-law form because of the action of various damping and dissipative processes. During several intermediate stages different components can have different spectra and, in some models, residual differences can still be present at the onset of non-linear stages.

In many respects the problem of explaining structure formation in the gravitational instability picture can be reduced to that of finding a power spectrum whose primordial form matches the COBE-inferred amplitude on large scales, and whose evolved form simultaneously matches the statistical properties of galaxies and clusters on smaller scales. This has proved to be a non-trivial task for the Cold Dark Matter (CDM) model, which, at least in its standard formulation $\left(\Omega_{0}=1, n=1, h=0.5\right.$ and Gaussian adiabatic fluctuations), is now generally accepted to be ruled out by the data (Wright et al. 1992; Taylor \& Rowan-Robinson 1992; Liddle \& Lyth 1993; White, Efstathiou \& Frenk 1993). The essential problem of this model is that, once it is normalized to match the measure CMB temperature anisotropy on large scales, it has too large a fluctuation amplitude on small scales $\lesssim 10 h^{-1} \mathrm{Mpc}$. Despite its failure, CDM is nevertheless considered as a reference model, several modifications of it having been suggested in order to remedy its shortcomings. Among the "second generation" of CDM-based models is the Cold+Hot Dark Matter (CHDM) model, which assumes that part of the dark matter content is in the form of massive neutrinos of mass $m_{\nu} \sim 10 \mathrm{eV}$ (Valdarnini \& Bonometto 1985; Bonometto \& Valdarnini 1985; Achilli, Occhionero \& Scaramella 1985; Holtzman 1989; Schaefer, Shafi \& Stecker 1992; Schaefer \& Shafi 1992; Davis, Summers \& Schlegel 1992; Holtzman \& Primack 1993; Liddle \& Lyth 1993; Klypin et al. 1993). In this scenario, the small-scale power is suppressed by neutrino free-streaming by an amount 
which depends on the hot percentage; the number of neutrino species participating in the hot component also plays a role (Primack et al. 1995; Babu, Shafer \& Shafi 1995). This model, with $\sim 20-30 \%$ to the density from the hot component appears to provide a good description of structure on a rather large range of scales, although stringent constraints on the exact amount of the hot component are provided by the abundance of high-redshift objects (e.g. Ma \& Bertschinger 1994; Klypin et al. 1995).

In this paper we discuss a scenario similar to the CHDM picture, where dark matter has a cold component (CDM) and a further volatile component (VDM), which has a phase space distribution resulting from the decay of a heavier particle species. A previous paper (Pierpaoli \& Bonometto 1995, hereafter PB95) calculated the effects of such a particle species upon the evolution of fluctuations through the period of recombination and up to the present epoch. Results were presented in PB95 in the form of transfer functions for several examples. In the following analysis we consider different models with respect to those discussed in PB95, so as to provide a wider sampling of the cold+volatile dark matter (CVDM) model parameter space. Furthermore, we go beyond the calculation of the transfer functions and submit the models to a number of explicit tests, by comparing them with observational data. The tests performed take into account: (i) the large-scale galaxy clustering as deduced from the analysis of volume-limited galaxy samples obtained from redshift catalogues; (ii) the behaviour of bulk velocities calculated using POTENT; (iii) the observed abundance of galaxy clusters; (iv) the observed abundance of high-redshift structures traced by damped Ly $-\alpha$ systems. Such tests therefore refer to scales ranging from a hundred of Mpc's down to a fraction of Mpc.

The plan of the paper is as follows. In Section 2 we introduce the CVDM models and the corresponding linear power spectra. By only resorting to linear-theory approaches, we compare in Section 3 such models to observational data. In Section 4 we discuss the main results and draw general conclusions from our analysis.

\section{The models}

The model we have in mind is one with two dark matter components, as well as baryonic material. To restrict the possible parameter space we consider only models with $\Omega_{\circ}=1$, $H_{0}=50 \mathrm{~km} \mathrm{~s}^{-1} \mathrm{Mpc}^{-1}$. As for the baryonic contribution, we consider the two values $\Omega_{b}=$ 0.05 and $\Omega_{b}=0.08$, which correspond to the central prediction of standard nucleosynthesis and to the $95 \%$ upper limit allowed by this constraint (e.g. Reeves 1994) given our chosen value of $H_{0}$.

In conventional Cold + Hot DM models, the hot component is assumed to be made of 
neutrinos with a mass of a few $\mathrm{eV}$ produced in thermal equilibrium in the early universe. In such a picture the redshift at which the neutrino becomes non-relativistic is $z_{n r} \simeq 1.4 \times$ $10^{4}\left(m_{\nu} / 10 \mathrm{eV}\right)$ and the contribution of $\nu$ 's to $\Omega_{o}$ is $\Omega_{\nu} \simeq 0.21 g_{\nu}\left(m_{\nu} / 10 \mathrm{eV}\right)\left(g_{\nu}\right.$ is the number of neutrino spin states with mass $m_{\nu}$, originally in thermal equilibrium). Accordingly $z_{n r} \simeq$ $6.7 \times 10^{4} \Omega_{\nu} / g_{\nu}$ and therefore $z_{n r}$ and $\Omega_{\nu}$ are not independent, as $g_{\nu}$ takes only suitable discrete values.

In the scenario we discuss here the VDM particles are produced by the decay of a more massive particle and are consequently never in thermal equilibrium. For the purposes of this paper, this means effectively that the parameters $z_{n r}$ and $\Omega_{X}$, where $X$ denotes the VDM species, can be varied independently.

It is also important to stress that there exist microphysical models where decay processes do indeed give rise to a cosmological scenario of the kind we discuss here. One such model is described by Bonometto, Gabbiani \& Masiero (1994) and discussed also in PB95. In this model both supersymmetry (SUSY) and Peccei-Quinn symmetries hold, and it is possible that the lightest eigenstate of the ordinary neutral fermion partners in SUSY theories, called the neutralino, can decay into the supersymmetric partner of the axion (called the axino). The non-thermal axinos produced by the decays would be VDM. Plausible values of the model parameters are $\Omega_{X}$ in the range 0.1 to 1.0 and $z_{n r}$ in the range $10^{3}$ to $10^{5}$. There does remain a constraint between $\Omega_{X}$ and the number of massless neutrinos which is imposed by primordial nucleosynthesis considerations (see PB95). We have mentioned this specific model merely as an illustration; others can be constructed, but their discussion is beyond the scope of this paper.

Whatever the origin of a non-thermal volatile component, it has a significant effect on the nature of the evolved fluctuation spectrum. Our criterion to select models in the $\Omega_{X}-z_{n r}$ parameter space can be sketched as follows. Let $g^{*}$ be the number of helicity states which are allowed by nucleosynthesis and $g_{\nu}$ that associated to neutrinos which are present at the nucleosynthesis epoch. Then $g_{X}=g^{*}-g_{\nu}$ is the number of extra spin states to be associated to VDM particles. If $z_{n r}$ is the redshift at which VDM becomes non relativistic, then the limit for $\Omega_{X}$ reads

$$
\Omega_{X} \lesssim \frac{z_{n r}}{10^{5}} g_{X}
$$

(see PB95). For each $\Omega_{X}$, we choose three different $z_{n r}$ values, namely $z_{n r}=2 \times 10^{4} \Omega_{X}, 5 \times$ $10^{4} \Omega_{X}$, and $2 \times 10^{5} \Omega_{X}$.

According to Walker et al. (1991), standard stellar light element abundances, combined with nucleosynthesis calculations, allow up to $g^{*}=7$, a value which is also quite close to the $2 \sigma$ upper bound provided by Olive and Scully (1995). Note, however, that Hata et al. (1995) recently gave the more stringent constraint $g^{*}<5$ at $95 \%$ C.L., which even conflicts 
with the usual 3 neutrino species. In this paper, we adopt the limit $g^{*}=7$.

The first value of $z_{n r}$ given above corresponds to $g_{X}=5$, if eq.(1) holds as an equality. Accordingly, the number of allowed neutrino species is $N_{\nu}=g_{\nu} / 2=1$. This implies that only one massless neutrino is present at nucleosynthesis; the remaining two neutrinos are quite heavy and have been already decayed. Taking $z_{n r}=5 \times 10^{4} \Omega_{X}$ instead allows $g_{X}=3$ with $N_{\nu}=2$, while the choice $z_{n r}=2 \times 10^{5} \Omega_{X}$ allows one helicity state for VDM particles with $N_{\nu}=3$.

Models with the first value of $z_{n r}$ would thus be inconsistent with the Hata et al. (1995) limits. But note also that, contrary to what one might naively expect, the results we quote below for higher values of $z_{n r}$ cannot be used straightforwardly to accomodate this more stringent constraint because the shape of the transfer function depends explicitly on the number of massless neutrino species.

As for $\Omega_{X}$, we choose values in the interval $0.1 \leq \Omega_{X} \leq 0.5$, with step 0.1 . For each value of $\Omega_{X}$ we allow for both the above baryonic fractions. In Table 1 we list the parameters for the resulting 30 models, on which we base the discussion of this paper. Note that, with these choices for $z_{n r}$ and $\Omega_{X}$, differ substantially from those presented in PB95 and probe a more carefully chosen part of the parameter space.

For each model, we follow the evolution of the fluctuations in the baryonic $\left(\delta_{b}\right)$, cold $\left(\delta_{c}\right)$ and volatile $\left(\delta_{X}\right)$ components through equivalence and recombination epochs (the algorithm used here has drastically reduced the CPU times with respect to the one used in PB95; see however PB95 for its details). The transfer function is, a priori, different for the various components. Since we are not interested here in following the evolution of each single component, in what follows we define the overall transfer function as the ratio

$$
T(k)=\frac{\left(\delta_{b}+\delta_{c}+\delta_{X}\right)_{z=0}}{\left(\delta_{b}+\delta_{c}+\delta_{X}\right)_{z \gg z_{\mathrm{hor}}}} .
$$

Accordingly, $T(k)$ is normalized to unity at scales so large that they enter the horizon after recombination. In the above definition, $z_{\text {hor }}$ is a redshift at which the smallest scale considered is still well above the horizon scale. Numerical values of $T(k)$ are provided by the parametric expression

$$
T(k)=\left(1+\sum_{j=1}^{4} c_{j} k^{j / 2}\right)^{-1},
$$

where the $c_{j}$ generally depend on the redshift, due to the residual free-streaming of volatile particles. The values of the fitting parameters at $z=0$ are given in Table 1 , with $k$ measured in $\mathrm{Mpc}^{-1}$. The limiting scale down to which transfer functions are computed is $250 \mathrm{kpc}$. 
We will not go into a detailed analysis of the relative behaviour of the models here, but it is worth pointing out a couple of important trends. Firstly, at fixed $z_{n r}$ and $N_{\nu}$, the amount of small scale power relative to large decreases as $\Omega_{X}$ is increased, due to the progressively larger effect of free-streaming. On the other hand, at fixed $N_{\nu}$ and $\Omega_{X}$, the power on small scales increases relative to large scales as $z_{n r}$ is increased, since a larger $z_{n r}$ corresponds to smaller velocities at the present time, and a consequent reduction of the effects of free-streaming.

Assuming that the COBE signal contains a negligible contribution from gravitational waves (see, e.g., Lidsey \& Coles 1992), the spectrum normalization can be accomplished by matching the observed COBE fluctuation spectrum with its predicted form:

$$
C_{l}=\left\langle\left|a_{l m}\right|^{2}\right\rangle=\frac{1}{2 \pi}\left(\frac{H_{0}}{c}\right)^{4} \int_{0}^{\infty} P(k) j_{l}^{2}\left(k x_{H}\right) k^{-2} d k,
$$

where $j_{l}$ is a spherical Bessel function and $x_{H}=2 c / H_{0}$. We take $P(k)=A k$ (scale-free primordial fluctuations: Harrison 1970; Zel'dovich 1972). The spectrum amplitude $A$ is estimated by matching the quadrupole $(l=2)$ value of $Q_{\mathrm{rms}-\mathrm{PS}}=20 \mu \mathrm{K}$ (Gorski et al. 1994; Wright et al. 1994), with an approximate $10 \%$ uncertainty on this value (Gorski, Stompor \& Banday 1995). The value of $A$ required to normalize each model in this way is also displayed in Table 1.

\section{Observational tests}

We concentrate here on comparing analytical predictions of the CVDM models with observational constraints on large-scale properties of the density and velocity fields, as well as on abundances of cosmic structures. More refined investigations involving the use of $\mathrm{N}$-body simulations to account properly for effects of non-linear clustering, are beyond the scope of this paper. All the results in the following depend only on the linear power-spectrum. Results for the CVDM models are also compared to the predictions of the standard CDM and the CHDM model with $\Omega_{\nu}=0.3$ for the fractional density contributed by one massive neutrino species.

\subsection{Power-Spectra}

In order to compare the power spectra of the CVDM models to that of the galaxy distribution, we plot in Figure $1 P(k)$ for models with $\Omega_{X}=0.1,0.2,0.3$ and 0.5 , as well as for two volumelimited subsamples of the CfA2 and SSRS2 redshift surveys (da Costa et al. 1994). The 
spectra plotted are all normalized to COBE, so that galaxies are assumed to trace exactly the DM distribution (allowing for linear biasing would just result in a vertical shift of the curves). Linear-theory redshift space distortions are introduced by multiplying $P(k)$ by the correction factor $f=1+2 /(3 b)+1 /\left(5 b^{2}\right)$ (Kaiser 1987), where we assume $b=1$ for the biasing factor. Since we plot linear spectra, we should take in mind that the comparison with data is reliable only for $k \lesssim 0.2\left(h^{-1} \mathrm{Mpc}\right)^{-1}$. At smaller scales, non-linear gravitational clustering makes both the linear spectrum shape and the treatment for redshift-space distortions inadequate. In any case, we expect the net result of non-linear effects to be an increase of $P(k)$. Those models whose linear $P(k)$ already fall above the data points would therefore have even harder time if a proper non-linear treatment were performed; this is the case for CDM and for all the $\Omega_{X}=0.1$ CVDM models. As $\Omega_{X}$ is increased, we note that the models with large values of $z_{n r}$ display more power than the data for $0.1 \lesssim k \lesssim 0.2\left(h^{-1} \mathrm{Mpc}\right)^{-1}$. This is just the consequence of the fact that, at larger values of $z_{n r}$ the volatile particles have smaller velocities and, therefore, fall into the CDM potential wells at an earlier epoch.

In order to provide a more quantitative description of the clustering, we compute $\sigma_{8}$, which is defined as the rms fluctuation amplitude,

$$
\sigma_{R}=\left[\frac{1}{2 \pi^{2}} \int_{0}^{\infty} P(k) W^{2}(k R) k^{2} d k\right]^{1 / 2},
$$

within a top-hat window, $W(k R)=3(\sin k R-k R \cos k R) /(k R)^{3}$, of radius $R=8 h^{-1} \mathrm{Mpc}$. The $\sigma_{8}$ values for all the models are listed in Table 2 and are compared with that, $\sigma_{8}=$ $0.90 \pm 0.05$, reported by Loveday et al. (1995) for Stromlo-APM galaxies in real space. It turns out that models with $\Omega_{X}=0.1$ or with large $z_{n r}$ have the unpleasant feature of implying a substantially antibiased galaxy distribution, in some cases comparable to that of CDM. A constraint on $\sigma_{8}$ for the DM distribution comes from the cluster abundance, which suggests $\sigma_{8} \simeq 0.6$ for $\Omega_{0}=1$ models (e.g. White, Efstathiou \& Frenk 1993; see below for a more detailed discussion of the cluster abundance predictions for our models). We note that only small variations of $\sigma_{8}$ are obtained by increasing the baryonic contribution from $5 \%$ to $8 \%$.

Smaller values of $\sigma_{8}$ may be allowed by the COBE normalisation if one tilts the primordial spectral index to $n<1$, also possibly allowing for the presence of some tensor contribution in the CMB temperature anisotropies. For instance, taking $n=0.9$ for the model $4\left(\Omega_{X}=0.2\right.$, $z_{n r}=4 \times 10^{3}$ ) one gets $\sigma_{8}=0.70$.

As a further characterization of the power-spectrum shape, we computed the parameter $\Gamma$ defined by $\Gamma=0.5\left(3.4 \sigma_{25} / 0.95 \sigma_{8}\right)^{-1 / 0.3}$ (Wright et al. 1992; Efstathiou, Bond \& White 1992); $\Gamma \simeq 0.25$ is required by the data. According to the values reported in Table 2 , we confirm the visual impression obtained from Figure 1: models with $\Omega_{X}=0.1$ have a too large 
$\Gamma$ (they are too similar to CDM), while models with a larger amount of volatile component fare better, unless one takes a large value of $z_{n r}$.

\subsection{Bulk Velocities}

The rms bulk velocity, $V_{\text {bulk }}(R)$, is defined as the rms matter velocity after smoothing over a volume of size $R$. For $\Omega_{\circ}=1$ it is connected to the power-spectrum according to

$$
V_{\mathrm{bulk}}^{2}(R)=\frac{H_{0}^{2}}{2 \pi^{2}} \int_{0}^{\infty} P(k) W^{2}(k R) d k,
$$

where $W(k R)$ is the window function specifying the shape of the smoothing volume. By comparing eqs.(5) and (6), it is clear that $V_{\text {bulk }}(R)$ gives more weight to long wavelength modes than than $\sigma_{R}$. Therefore, we expect bulk velocities on large scales to depend only on the $Q_{r m s-P S}$ normalization and not on the profile of the transfer function.

Reliable $V_{\text {bulk }}$ data for top-hat spheres centered on the Local Group on scales of few tens of Mpcs are provided by the POTENT reconstruction method (e.g. Bertschinger et al. 1990; see also Dekel 1994, and references therein). In Figure 2 we compare our model predictions with the latest POTENT data (courtesy of A. Dekel). In order to account for the velocity smoothing procedure in the reconstruction method, we convolved the powerspectrum in eq.(6) with a Gaussian filter of radius $R_{f}=12 h^{-1} \mathrm{Mpc}$. As expected, any difference between models at large scales is negligible and all of them are in remarkable agreement with data. On smaller scales $\left(\lesssim 40 h^{-1} \mathrm{Mpc}\right)$ the predicted $V_{\text {bulk }}$ values tend to be larger than the observational one. In this respect, models with $\Omega_{X} \geq 0.4$ perform better, thanks to the steep $P(k)$ profile at large $k$, although this is unfortunately inconsistent with galaxy clustering data. On the other hand, independent estimates of bulk flows (e.g. da Costa et al. 1995) agree with the POTENT one only on scales $R \simeq 50-60 h^{-1} \mathrm{Mpc}$. Therefore, we do not regard this marginal discrepancy as a serious problem for any of the models we have considered.

\subsection{Cluster abundance}

According to the standard Press \& Schechter (1974) approach, the number density of collapsed structures arising from Gaussian initial fluctuations and having mass larger than $M$ is given by

$$
N(>M)=\int_{M}^{\infty} n\left(M^{\prime}\right) d M^{\prime}
$$


Here $n(M) d M$ is the number density of objects with mass in the range $[M, M+d M]$ and is related to the power-spectrum according to

$$
n(M) d M=\frac{1}{\sqrt{2 \pi}} \frac{\delta_{c}}{f} \int_{R}^{\infty} \frac{\eta_{R}}{\sigma_{R}} \exp \left(-\frac{\delta_{c}^{2}}{2 \sigma_{R}^{2}}\right) \frac{d R}{R^{2}},
$$

where

$$
\eta_{R}=\frac{1}{2 \pi^{2} \sigma_{R}^{2}} \int k^{4} P(k) \frac{d W^{2}(k R)}{d(k R)} \frac{d k}{k R} .
$$

In the above expressions, we assume that the mass scale $M$ is related to the length scale $R$ according to $M=f \bar{\rho} R^{3}$, with $f$ the "form factor", which is specified by the shape of the filter $W$ and $\bar{\rho}$ the average density. For the Gaussian window, that we assume here, it is $f=(2 \pi)^{3 / 2}$, while $f=4 \pi / 3$ for a top-hat window. The parameter $\delta_{c}$ is the critical density contrast, which represents the threshold value for a fluctuation to turn into an observable object, if evolved to the present time by linear theory. For a top-hat spherical collapse one has $\delta_{c}=1.68$, but the inclusion of non-linear effects, as well as aspherical collapse, may lead to a lower value of $\delta_{c}$. For example, Klypin \& Rhee (1994; KR94 hereafter) found that the cluster mass function in their CHDM N-body simulations is well fit by eq.(8) by taking $\delta_{c}=1.5$ for the Gaussian window. In order to account for the rather poor knowledge of $\delta_{c}$, we prefer to compute $N(>M)$ for different values of this parameter in the range $[1.4,1.7]$.

The results of this analysis are reported in Figure 3, where we also compare them with observational results. Values of $N(>M)$ for $\delta_{c}=1.5$ are also listed in Table 2. Following White et al. (1993), we take $M=4.2 \times 10^{14} h^{-1} M_{\odot}$ for the limiting mass at which to estimate the mass function. We prefer not to consider a larger value, $\sim 10^{15} h^{-1} M_{\odot}$, since this would correspond to the exponential tail of the cluster mass function (e.g. Bahcall \& Cen 1992) and, as a consequence, large variations in the cluster abundance would be associated with uncertainties in the cluster mass estimates. The dashed band in Figure 3 corresponds to the range between the observational result of White et al. (1993; lower limit) based on $X$-ray data, and that of Biviano et al. (1993; upper limit) based on velocity dispersions. We note that realistic observational uncertainties are probably larger than the difference between such two results. They may be due to systematic effects, related to assumptions used to connect $X$-ray temperature and DM potential profiles, or to biases in estimating cluster masses from internal velocities under the virial assumption.

Even bearing such warnings in mind, it seems difficult to reconcile with the data those models which overproduce clusters by one order of magnitude or more, for any value of $\delta_{c}$ value. This is the case for $\Omega_{X}=0.1$ and, in general, for those models having a large value of $z_{n r}$. Even though taking the larger baryonic fraction decreases the cluster abundance, 
its effect is nevertheless only marginal for those models which have an exceedingly large $N(>M)$.

It is not clear whether such large discrepancies may be overcome on the ground of observational biases. For instance, let us consider the model $5\left(\Omega_{X}=0.2, z_{n r}=10^{4}\right)$ as a case providing a large $N(>M)$. If we allow for an underestimate of cluster masses by a factor 2 (i.e., $M=8.4 \times 10^{14} h^{-1} M_{\odot}$; see, however, Evrard, Metzler \& Navarro 1995, for arguments in favour of precise mass determinations from $X$-ray data) and take $\delta_{c}=1.5$, it would give $N(>M) \simeq 1.2 \times 10^{-5}\left(h^{-1} \mathrm{Mpc}\right)^{-3}$, which is still quite far from the observational band.

On the other hand, we do not believe that the observational situation is clear enough to rule out at a large confidence level models which are discrepant by a factor 2-3 with respect to the reported abundances. Even adopting such a rather conservative position, it is fair to say that only models with $\Omega_{X} \geq 0.2$ and low $z_{n r}$ are not excluded by this constraint. In this respect, the availability of more and more determinations of cluster masses based on the independent technique of weak gravitational lensing (e.g. Squires et al. 1995) will be extremely welcome.

\subsection{High-redshift behaviour}

A further constraint on power spectra comes from observations of high-redshift objects. The most reliable such constraint concerns the abundance of damped Ly- $\alpha$ systems (DLAS). These are observed as wide absorbtion troughs in quasar spectra, due to a high HI column density $\left(\geq 10^{20} \mathrm{~cm}^{-2}\right)$. The fact that at $z \sim 3$ the fractional density of HI gas associated with DLAS is comparable to that contributed by visible matter in nearby galaxies, suggests that DLAS actually trace a population of collapsed protogalactic objects (see Wolfe 1993, for a comprehensive review). Lanzetta et al. (1995) and Wolfe et al. (1995) presented data on DLAS up to the redshift $z \simeq 3.5$, while the recent compilation by Storrie-Lombardi et al. (1995) pushed this limit to $z \simeq 4.25$. Based on these data, the latter authors claimed the first detection of a turnover in the fractional density, $\Omega_{g}$, of neutral gas belonging to the absorbing systems at high redshift.

Several authors recognized DLAS as a powerful test for DM models, based on a linear theory approach, Subramanian \& Padmanabhan (1994), Kauffman \& Charlot (1994), Mo \& Miralda-Escudé (1994) and Ma \& Bertschinger (1994) concluded that the standard CHDM scenario with $\Omega_{\nu}=0.3$ is not able to generate enough collapsed structures at $z \gtrsim 3$, due to the lack of power on galactic scales. However, either lowering $\Omega_{\nu}$ to about 0.2 (Klypin et al. 1995) or 'blueing' the primordial spectrum, $P_{i}(k) \propto k^{n}$ to $n \simeq 1.2$ (Borgani et al. 1995) keeps CHDM into a better agreement with data. Katz et al. (1995) resorted to numerical simulations of DLAS and found that even the CDM model with a normalization as low as 
$\sigma_{8}=0.7$ satisfies the DLAS constraint.

In order to connect model predictions from linear theory with observations, let

$$
\Omega_{\text {coll }}(M, z)=\operatorname{erfc}\left(\frac{\delta_{c}}{\sqrt{2} \sigma(M, z)}\right),
$$

be defined as the fractional density contributed at the redshift $z$ by collapsed structures of mass larger than $M$. Accordingly, it is $\Omega_{g}=\alpha_{g} \Omega_{b} \Omega_{\text {coll }}$, where $\alpha_{g}$ is the fraction of HI gas which is involved in the absorbers.

One expects the value of $\alpha_{g}$, to decrease well below unity at low redshift, due to gas consumption into stars. Note that varying this number turns into a linear rescaling of $\Omega_{g}$. Since we assume here that $\alpha_{g}=1$, we compare data and CVDM predictions at the highest redshift allowed by the data, $z=4.25$ (Storrie-Lombardi et al. 1995). We estimate $\sigma(M, z=4.25)$ for a Gaussian window, by explicitly computing the transfer function at that redshift, so as to take into account effects of residual free-streaming of the volatile component.

As for the value of the critical density contrast $\delta_{c}$, results based on $\mathrm{N}$-body approaches by Efstathiou \& Rees (1988) and by Klypin et al. (1995) suggests that $1.3 \lesssim \delta_{c} \lesssim 1.5$ for a Gaussian window, while Ma \& Bertschinger (1994) found indications for $\delta_{c} \simeq 1.7-1.8$ for a top-hat window. In the following we will report results for $\delta_{c}=1.5$ and gaussian window; while the effect of varying this parameter is discussed in more detail by Borgani et al. (1995). Lacey \& Cole (1994) have realized a detailed test of the Press-Schechter theory against scale-free $\mathrm{N}$-body simulations, by checking the effects of varying the window and the halo identification method. As a result, they found that $\delta_{c} \simeq 1.3$ and $\delta_{c} \simeq 1.8$ are in general adequate to describe the halo mass function for Gaussian and top-hat window, respectively. In any case, we verified that rather similar results are obtained either using $\delta_{c}=1.5$ with a Gaussian window, or $\delta_{c}=1.7$ with a top-hat window.

The results of our analysis are reported in Figure 4, where we plot the neutral gas fraction associated to DLAS, $\Omega_{g}$, for all the models with $\Omega_{b}=0.05$ and compare them with the observational data. In the light of all the above-mentioned uncertainties in realising such a comparison, we prefer here to adopt a conservative approach and to consider in this comparison the result of Storrie-Lombardi et al. (1995; $\Omega_{g}=2.2 \pm 0.5$ at $z=4.25$ for $\Omega_{0}=1$ and $h=0.5$ ) as a lower bound. Consistently, the dashed areas in Figure 4 are delimitated by the above $1 \sigma$ lower limit. Only model falling below this limit are ruled out. The effect of varying the limiting mass of the protogalaxy hosting DLAS by an order of magnitude may be judged by comparing open and filled dots, which correspond to $M=5 \times 10^{9} h^{-1} M_{\odot}$ and $M=5 \times 10^{10} h^{-1} M_{\odot}$, respectively. Numerical values of $\Omega_{g}$ for all the models are reported in column 5 of Table 2, where also results for CDM and CHDM are given. 
As expected, taking lower $\Omega_{X}$ and larger $z_{n r}$ make easier the agreement with data. All the models with $\Omega_{X} \leq 0.2$ are able to pass the DLAS test, while larger volatile fractional densities are only allowed for large $z_{n r}$, which however turns into a wrong power-spectrum shape.

Although several models can clearly be ruled out already at this level on the ground of DLAS data, nevertheless it is clear that more precise conclusions can only be reached with a better knowledge of the variables entering in the Press-Schechter prediction for $\Omega_{g}$ (i.e. the mass $M$, and the parameters $\delta_{c}$ and $\alpha_{g}$ ). A more accurate definition of what is a DLAS in a given DM model can only be achieved with numerical simulations involving hydrodynamics (Katz et al. 1995), which would be able to trace the history of galaxy formation. As for observations, the possibility that systematic biases may affect the final results have been recently suggested. For instance, Bartelmann \& Loeb (1995) have recently pointed out that amplification biases due to gravitational lensing of QSOs by DLAS could led to an overestimate of $\Omega_{g}$, by an amount which however decreases with redshift. Fall \& Pei (1995) argued that dust obscuration may act in the opposite direction so as to bias downwards the estimated $\Omega_{g}$. Verifying the actual relevance of such effects surely requires a substantial investment of observational and theoretical effort.

\section{Discussion}

The results we have presented demonstrate that the CVDM hypothesis yields potentially interesting models of structure formation. The aim of this paper is to show that rather slight changes in the parameters of volatile dark matter can make a significant difference to the transferred power spectrum. This contrasts with the case of a cold component, where the physical properties of the candidate particle do not really matter at all, in that the physical origin of the hot particles may leave a detectable imprint in the clustering pattern. In this context it is important to verify up to which point the shape of the distribution function causes differences compared to the standard scenario based on relic thermal neutrinos.

The ability to change $z_{n r}$ almost independently of $\Omega_{X}$ is especially significant in this respect: the power spectra we have obtained display considerable variations at a fixed value of $\Omega_{X}$. Indeed, although the CVDM class of models involves one more parameter than is the case for CHDM, we have shown that observational data nevertheless allow us to put rather stringent constraints on the permitted values of $z_{n r}$ and $\Omega_{X}$, even at the level of lineartheory. The most stringent of these constraints comes from the simultaneous requirement for a model to satisfy the observed abundance of high-redshift DLAS and of galaxy clusters. As for DLAS, the rather large value of the HI gas fraction involved in the absorbing systems, 
$\Omega_{g}$, implies a substantial amount of power on galaxy scales, so as to favour models with $\Omega_{X} \lesssim 0.2$. A larger volatile component would be allowed only resorting to a high value of $z_{n r} \simeq 2 \times 10^{5} \Omega_{X}$ (cf. Figure 4). On the other hand, models with small $\Omega_{X}$ and/or large $z_{n r}$ behave too much like the standard CDM model, drastically overproducing clusters (cf. Figure 3).

Therefore, the overall result would be that models with $z_{n r} \gtrsim 5 \times 10^{4} \Omega_{X}$ have a hard time, quite independently of $\Omega_{X}$. Among the models inspected, the only model which passes all the tests, or at least which can not be confidently ruled out, is the one with $\Omega_{X}=0.2$ and $z_{n r}=4 \times 10^{3}$. It is worth recalling, however, that such a model with low $z_{n r}$ requires that volatile particles occupy at least 5 helicity states [cf. eq.(1)]. We recall that this can accommodated only if (a) $g^{*}=7$ is allowed by standard nucleosynthesis and (b) two neutrino species are sufficiently massive that they have already decayed at the nucleosynthesis epoch.

An alternative possibility, holding if the physics of the decay is quite different from the axino model suggested in PB95, is that the decay itself takes place after the nucleosynthesis epoch. This would make low $z_{n r}$ models compatible with all $N_{\nu}$. It must, however, be remembered that changing $N_{\nu}$ itself causes an alteration of the transfer function, and a straightforward extrapolation of the above results to greater $N_{\nu}$ values is not allowed. Changing the relation between $N_{\nu}$ and $z_{n r}$ opens the way to inspecting different models and, in this context, we should also bear in mind that our analysis has been based on assuming a scale-free primordial spectrum, while variations around this model are allowed by some classes of inflationary schemes. For instance, taking $P_{i}(k) \propto k^{n}$ with $n<1$ (Adams et al. 1993; Liddle \& Lyth 1993 and references therein) decreases the amount of power on the cluster mass scale, so as to alleviate the problem of cluster overproduction displayed by "colder" models. However, the amount of this tilt can not be too large, in order not to conflict with CMB (Bennet et al. 1994) and large-scale peculiar motions constraints (Tormen et al. 1993). On the other hand, the case of "antitilting", with $n \simeq 1.2$, has been recently advocated to alleviate some of the problems of the CHDM scenario (Dvali, Shafi \& Schaefer 1994; Lucchin et al. 1995). However, the subsequent increase of power on small scales goes in the undesired direction as far as the cluster abundance is concerned (Pogosyan \& Starobinsky 1995; Borgani et al. 1995).

As a final remark, we should stress that the analysis presented in this paper is only preliminary and is entirely based on linear calculations. In order to be more definitive we would like to extend it in two main directions. Firstly to calculate more detailed properties of the CMBR fluctuations they produce: we anticipate a rather different signature on angular scales around a degree than in the standard CHDM models. Furthermore, we would also like to study the non-linear evolution of some of these model by performing numerical calculations using $\mathrm{N}$-body and other procedures. 


\section{Acknowledgements}

We wish to thank Michael Vogeley for providing us with the electronic version of the CfA2+SSRS2 power spectrum. The authors acknowledge the use of the QMW starlink facilities during this work. Elena Pierpaoli acknowledges financial support under the EC Human Capital \& Mobility Network 'Large Scale Structure in the Universe: Evolution and Statistics' (Contract No: ERBCHRX-CT93-0129). Stefano Borgani has been partially supported by Italian MURST. Peter Coles is a PPARC Advanced Research Fellow. We are extremely grateful to Carlton Baugh for his help with a preliminary version of this paper. 


\section{References}

Achilli S., Occhionero F., Scaramella R., 1985, ApJ, 299, 577

Adams F.C., Bond J.R., Freese K., Freeman J.A., Olinto A.V., 1993, Phys. Rev. D, 47, 426

Babu K.S., Schaefer R.K., Shafi Q., 1995, preprint astro-ph/9507006

Bahcall N.A., Cen R, 1992, ApJ, 398, L1

Bartelmann M., Loeb A., 1995, ApJ, submitted, preprint astro-ph/9505078

Bahcall N.A., Cen R., 1992, ApJ, 398, L81

Bennet C.L., et al. 1994, ApJ, 430, 423

Bertschinger E., Dekel A., Faber S.M., Dressler A., Burstein D., 1990, ApJ, 364, 370

Biviano A., Girardi M., Giuricin G., Mardirossian F., Mezzetti M., 1993, ApJ, 41, L13

Bonometto S., Gabbiani F, Masiero A., 1994, Phys. Rev. D., 49, 3918

Bonometto S., Valdarnini R., 1985, ApJ, 299, L71

Borgani S., Lucchin F., Matarrese S., Moscardini L., 1995, MNRAS, in press, preprint astroph/9506003

da Costa L.N., Vogeley M.S., Geller M.J., Huchra J.P., Park C., 1994, ApJ, 437, L1

da Costa et al., 1995, in eds. Balkowski C., Maurogordato S., Tao C. \& Trân Thanh Vân

J., Proc. of the Moriond Astrophysics Meeting on Clustering in the Universe, in press

Davis M., Summers F.J., Schlegel D., 1992, Nat, 359, 393

Dekel A., 1994, ARA\&A, 32, 371

Dvali G., Shafi Q., Schaefer R.K., 1994, Phys. Rev. Lett. 73, 1886

Efstathiou G., Bond J.R., White S.D.M., 1992, MNRAS, 258, 1p

Efstathiou G., Rees M.J., 1988, MNRAS, 230, 5p

Evrard A.E., Metzler C.A., Navarro J.F., 1995, ApJ, preprint astro-ph/9510058

Fall S.M., Pei Y.C., 1995, in QSO absorbtion Lines. Springer Verlag, Berlin, in press

Gorski K.M. et al., 1994, ApJ, 430, L89

Gorski K.M., Stompor R., Banday A.J., 1995, preprint astro-ph/9502033

Holtzman J.A., 1989, ApJS, 71, 1

Holtzman J.A., Primack J.R., 1993, ApJ, 405, 428

Kaiser N., 1987, MNRAS, 227, 1

Katz N., Weinberg D.H., Hernquist L., Miralda-Escudé J., 1995, preprint astro-ph/95109106

Kauffmann G., Charlot S., 1994, ApJ, 430, L97

Klypin A., Holtzman J., Primack J.R., Regös E., 1993, ApJ, 415, 1

Klypin A., Borgani S., Holtzman J., Primack J.R., 1995, ApJ, 444, 1

Lacey C., Cole S., 1994, MNRAS, 271, 676

Lanzetta K.M, Wolfe A., Turnshek D.A., 1995, ApJ, 440, 435

Liddle A.R., Lyth D.H., 1993, Phys. Rep., 231, 1 
Lidsey J.E., Coles P., 1992, MNRAS, 258, 57p

Loveday J., Efstathiou G., Maddox S.J., Peterson B.A., 1995, ApJ, 442, 457

Lucchin F., Colafrancesco S., de Gasperis G., Matarrese S., Mei S., Mollerach S., Moscardini L., Vittorio N., 1995, ApJ, in press

Ma C.P., Bertschinger E., 1994, ApJ, 434, L5

Mo H.J., Miralda-Escudé J., 1994, ApJ, 430, L25

Olive K.A., Scully S.T., 1995, preprint astro-ph/9506131

Peacock J.A., Dodds S.J., 1994, MNRAS, 267, 1020

Pierpaoli E., Bonometto S.A., 1995, A\& A, 300, 13

Primack J.R., Holtzman J., Klypin A., Caldwell D.O., 1995, Phys. Rev. Lett., 74, 2160

Pogosyan D. Yu., Starobinsky A.A., 1995, ApJ, 447, 465

Press W.H., Schechter P.L., 1974, ApJ, 187, 425

Reeves H., 1994, Rev. Mode. Phys., 66, 193

Schaefer R.K., Shafi Q., 1992, Nat, 359, 199

Schaefer R.K., Shafi Q. Stecker F., 1989, ApJ, 347, 575

Smoot G.F. et al., 1992, ApJ, 396, L1

Squires G., Kaiser N., Babul A., Fahlman G., Woods D., Neumann D.M., Böhringer H., 1995, preprint astro-ph/9507008

Storrie-Lombardi L.J., McMahon R.G., Irwin M.J., Hazard C., 1995, in Proceedings of the ESO Workshop on QSO Absorbtion Lines, preprint astro-ph/9503089

Subramanian K., Padmanabhan T., 1994, preprint astro-ph/9402006

Taylor A.N., Rowan-Robinson M., 1992, Nat, 359, 396

Tormen G., Moscardini L., Lucchin F., Matarrese S., 1993, ApJ, 411, 16

Valdarnini R., Bonometto S., 1985, A\& A, 146, 235

van Dalen A., Schaefer R.K., 1992, ApJ, 398, 33

Walker T.P., Steigman G., Schramm D.N., Olive K.A., Kang H., 1991, ApJ, 376, 51

White S.D.M., Efstathiou G., Frenk C.S., 1993, MNRAS, 262, 1023

Wolfe A., 1993, in Relativistic Astrophysics and Particle Cosmology, eds. C.W. Ackerlof, M.A. Srednicki, (New York: New York Acad. Sci.), p.281

Wolfe A.M., Lanzetta K.M., Foltz C.B., Chaffee F.H., 1995, preprint

Wright E.L. et al., 1992, ApJ, 396, L9

Wright E.L., Smoot G.F., Bennett C.L., Lubin P.M., 1994, ApJ, 436, 443 


\section{Figure Captions}

Figure 1. Comparison between observational and CVDM linear power-spectra in redshift space. Open and filled triangles are the power-spectrum for two volume limited subsamples of the combined CfA2+SSRS2 survey (da Costa et al. 1994). Each panel refers to a fixed value of the volatile fraction $\Omega_{X}$, while the dotted, short-dashed and long-dashed curves correspond to different $z_{n r}$. For reference, we also plot the CDM (solid curves). All the models are for $\Omega_{b}=0.05$.

Figure 2. Comparison between the POTENT bulk flow (filled dots) and that provided by the CVDM models. Different panels are for different $\Omega_{X}$ values. In each panel, different curves refer to the different values for $z_{n r}$.

Figure 3. The abundance of galaxy clusters with $M>4.2 \times 10^{14} h^{-1} M_{\odot}$. The shaded area is delimited by the observational results by Biviano et al. (1993; upper limit) and by White et al. (1993; lower limit). In each panel, corresponding to different $\Omega_{X}$ values, dotted, short-dashed and long-dashed curves are for the three different values of $z_{n r}$. Each pair of curves correspond to the two values of $\Omega_{b}$, the lower one being for $\Omega_{b}=0.08$ and the higher one for $\Omega_{b}=0.05$.

Figure 4. The fractional density of neutral gas involved in DLAS at redshift $z=4.25$. The shaded area is the observational constraint and is delimited from below by the $1 \sigma$ lower limit by Storrie-Lombardi et al. (1995). Each panel refers to a fixed $\Omega_{X}$ value and reports the predicted $\Omega_{g}$ as a function of $z_{n r}$. Filled and open dots correspond to $M=5 \times 10^{9} h^{-1} M_{\odot}$ and $M=5 \times 10^{10} h^{-1} M_{\odot}$ for the limiting mass of the protostructures hosting DLAS. We assume a Gaussian window with $\delta_{c}=1.5$ and $\alpha_{g}=1$ for the HI gas fraction involved in the absorber. 
Table 1: Model parameters and power spectra. Column 2: number of massless neutrino species; Column 3: volatile fractional density; Column 3: redshift at which the volatile component becomes non-relativistic (in units of $10^{4}$ ); Columns 5 to 9: fitting parameters of the transfer functions [see eq.(3)].

\begin{tabular}{|c|c|c|c|c|c|c|c|c|}
\hline Model & $\mathbf{N}_{\nu}$ & $\Omega_{\mathrm{X}}$ & $\mathrm{z}_{\mathrm{nr}} / 10^{4}$ & $A\left(10^{7}\right)$ & $\mathrm{c}_{1}$ & $\mathrm{c}_{2}$ & $\mathrm{c}_{3}$ & $\mathrm{c}_{4}$ \\
\hline \multicolumn{9}{|c|}{$\Omega_{b}=0.05$} \\
\hline 1 & 1 & 0.1 & 0.2 & 1.300 & $-0.6133 \mathrm{E}+00$ & $0.1494 \mathrm{E}+02$ & $0.1220 \mathrm{E}+03$ & $0.6733 \mathrm{E}+02$ \\
\hline 2 & 1 & 0.1 & 0.5 & 1.306 & $-0.4284 \mathrm{E}+00$ & $0.1212 \mathrm{E}+02$ & $0.9742 \mathrm{E}+02$ & $0.6607 \mathrm{E}+02$ \\
\hline 3 & 3 & 0.1 & 2.0 & 1.311 & $-0.3665 \mathrm{E}+00$ & $0.1542 \mathrm{E}+02$ & $0.7916 \mathrm{E}+02$ & $0.8917 \mathrm{E}+02$ \\
\hline 4 & 1 & 0.2 & 0.4 & 1.318 & $0.5110 \mathrm{E}-01$ & $-0.1016 \mathrm{E}+01$ & $0.1924 \mathrm{E}+03$ & $0.1559 \mathrm{E}+03$ \\
\hline 5 & 1 & 0.2 & 1.0 & 1.319 & $0.1032 \mathrm{E}-01$ & $0.4255 \mathrm{E}+01$ & $0.1113 \mathrm{E}+03$ & $0.1629 \mathrm{E}+03$ \\
\hline 6 & 3 & 0.2 & 4.0 & 1.327 & $0.4886 \mathrm{E}-02$ & $0.1624 \mathrm{E}+02$ & $0.3607 \mathrm{E}+02$ & $0.1997 \mathrm{E}+03$ \\
\hline 7 & 1 & 0.3 & 0.6 & 1.354 & $0.1263 \mathrm{E}+01$ & $-0.2246 \mathrm{E}+02$ & $0.2301 \mathrm{E}+03$ & $0.3903 \mathrm{E}+03$ \\
\hline 8 & 1 & 0.3 & 1.5 & 1.303 & $-0.5240 \mathrm{E}+00$ & $0.1509 \mathrm{E}+02$ & $0.2086 \mathrm{E}+02$ & $0.4061 \mathrm{E}+03$ \\
\hline 9 & 3 & 0.3 & 6.0 & 1.266 & $-0.1997 \mathrm{E}+01$ & $0.5180 \mathrm{E}+02$ & $-0.1305 \mathrm{E}+03$ & $0.4194 \mathrm{E}+03$ \\
\hline 10 & 1 & 0.4 & 0.8 & 1.325 & $0.1052 \mathrm{E}+00$ & $0.8104 \mathrm{E}+01$ & $-0.1939 \mathrm{E}+02$ & $0.1053 \mathrm{E}+04$ \\
\hline 11 & 1 & 0.4 & 2.0 & 1.248 & $-0.2475 \mathrm{E}+01$ & $0.5754 \mathrm{E}+02$ & $-0.2466 \mathrm{E}+03$ & $0.9205 \mathrm{E}+03$ \\
\hline 12 & 3 & 0.4 & 8.0 & 1.168 & $-0.5488 \mathrm{E}+01$ & $0.1240 \mathrm{E}+03$ & $-0.4753 \mathrm{E}+03$ & $0.8259 \mathrm{E}+03$ \\
\hline 13 & 1 & 0.5 & 1.0 & 1.249 & $-0.3115 \mathrm{E}+01$ & $0.1033 \mathrm{E}+03$ & $-0.7761 \mathrm{E}+03$ & $0.2781 \mathrm{E}+04$ \\
\hline 14 & 1 & 0.5 & 2.5 & 1.107 & $-0.7916 \mathrm{E}+01$ & $0.1846 \mathrm{E}+03$ & $-0.9825 \mathrm{E}+03$ & $0.2091 \mathrm{E}+04$ \\
\hline 15 & 3 & 0.5 & 10.0 & 1.063 & $-0.9233 \mathrm{E}+01$ & $0.1943 \mathrm{E}+03$ & $-0.8273 \mathrm{E}+03$ & $0.1325 \mathrm{E}+04$ \\
\hline \multicolumn{9}{|c|}{$\Omega_{b}=0.08$} \\
\hline 16 & 1 & 0.1 & 0.2 & 1.323 & $-0.1009 \mathrm{E}-01$ & $0.8960 \mathrm{E}+01$ & $0.1418 \mathrm{E}+03$ & $0.6768 \mathrm{E}+02$ \\
\hline 17 & 1 & 0.1 & 0.5 & 1.319 & $-0.2039 \mathrm{E}-01$ & $0.5736 \mathrm{E}+01$ & $0.1202 \mathrm{E}+03$ & $0.6457 \mathrm{E}+02$ \\
\hline 18 & 3 & 0.1 & 2.0 & 1.318 & $-0.6469 \mathrm{E}-01$ & $0.6622 \mathrm{E}+01$ & $0.1021 \mathrm{E}+03$ & $0.8145 \mathrm{E}+02$ \\
\hline 19 & 1 & 0.2 & 0.4 & 1.308 & $-0.4181 \mathrm{E}-01$ & $-0.9723 \mathrm{E}+01$ & $0.2449 \mathrm{E}+03$ & $0.1497 \mathrm{E}+03$ \\
\hline 20 & 1 & 0.2 & 1.0 & 1.348 & $0.9112 \mathrm{E}+00$ & $-0.1115 \mathrm{E}+02$ & $0.1681 \mathrm{E}+03$ & $0.1530 \mathrm{E}+03$ \\
\hline 21 & 3 & 0.2 & 4.0 & 1.317 & $-0.1257 \mathrm{E}+00$ & $0.1017 \mathrm{E}+02$ & $0.5465 \mathrm{E}+02$ & $0.1926 \mathrm{E}+03$ \\
\hline 22 & 1 & 0.3 & 0.6 & 1.443 & $0.3845 \mathrm{E}+01$ & $-0.5984 \mathrm{E}+02$ & $0.3617 \mathrm{E}+03$ & $0.3668 \mathrm{E}+03$ \\
\hline 23 & 1 & 0.3 & 1.5 & 1.346 & $0.7783 \mathrm{E}+00$ & $-0.3884 \mathrm{E}+01$ & $0.8075 \mathrm{E}+02$ & $0.3960 \mathrm{E}+03$ \\
\hline 24 & 3 & 0.3 & 6.0 & 1.203 & $-0.3672 \mathrm{E}+01$ & $0.6458 \mathrm{E}+02$ & $-0.1643 \mathrm{E}+03$ & $0.4436 \mathrm{E}+03$ \\
\hline 25 & 1 & 0.4 & 0.8 & 1.392 & $0.2294 \mathrm{E}+01$ & $-0.3311 \mathrm{E}+02$ & $0.1601 \mathrm{E}+03$ & $0.1005 \mathrm{E}+04$ \\
\hline 26 & 1 & 0.4 & 2.0 & 1.244 & $-0.2619 \mathrm{E}+01$ & $0.6079 \mathrm{E}+02$ & $-0.2866 \mathrm{E}+03$ & $0.1030 \mathrm{E}+04$ \\
\hline 27 & 3 & 0.4 & 8.0 & 1.159 & $-0.5641 \mathrm{E}+01$ & $0.1198 \mathrm{E}+03$ & $-0.4603 \mathrm{E}+03$ & $0.8270 \mathrm{E}+03$ \\
\hline 28 & 1 & 0.5 & 1.0 & 1.279 & $-0.2064 \mathrm{E}+01$ & $0.8336 \mathrm{E}+02$ & $-0.6956 \mathrm{E}+03$ & $0.2808 \mathrm{E}+04$ \\
\hline 29 & 1 & 0.5 & 2.5 & 1.148 & $-0.6521 \mathrm{E}+01$ & $0.1636 \mathrm{E}+03$ & $-0.1016 \mathrm{E}+04$ & $0.2456 \mathrm{E}+04$ \\
\hline 30 & 3 & 0.5 & 10.0 & 0.925 & $-0.1341 \mathrm{E}+02$ & $0.2297 \mathrm{E}+03$ & $-0.9345 \mathrm{E}+03$ & $0.1439 \mathrm{E}+04$ \\
\hline
\end{tabular}


Table 2: Statistical properties of large-scale structure. Column 2: r.m.s. fluctuations within a top-hat sphere of $8 h^{-1} \mathrm{Mpc}$ radius. The observational result refers to the APM galaxy distribution in real space (Loveday et al. 1995). Column 3: 'extra power' parameter (see text); observational result from Peacock \& Dodds (1994). Column 4: number density of clusters with mass larger than $M=4.2 \times 10^{14} h^{-1} M_{\odot}$ (in units of $\left.10^{-6}\left(h^{-1} \mathrm{Mpc}\right)^{-3}\right)$. Lower and upper values for the observational result are from White, Efstathiou \& Frenk (1993) and Biviano et al. (1993), respectively. Column 5: fractional density of neutral gas within collapsed structures of mass $510^{10} h^{-1} M_{\odot}$ at redshift $z=4.25$, in units of $10^{-3}$; the Gaussian window with $\delta_{c}=1.5$ is assumed; observational result from Storrie-Lombardi et al. (1995).

\begin{tabular}{ccccc}
\hline \hline Model & $\sigma_{\mathbf{8}}$ & $\boldsymbol{\Gamma}$ & $N(>M)$ & $\boldsymbol{\Omega}_{\mathrm{g}}$ \\
\hline Observ. & $0.90 \pm 0.05$ & $0.25 \pm 0.05$ & $(4-6)$ & $2.2 \pm 0.5$ \\
& & & & \\
& & $\Omega_{b}=0.05$ & & \\
1 & 1.05 & 0.30 & 36 & 8.7 \\
2 & 1.23 & 0.34 & 53 & 11 \\
3 & 1.23 & 0.35 & 53 & 10 \\
4 & 0.87 & 0.19 & 19 & 1.4 \\
5 & 1.10 & 0.25 & 40 & 3.2 \\
6 & 1.23 & 0.33 & 53 & 5.1 \\
7 & 0.76 & 0.13 & 11 & $4 \mathrm{E}-02$ \\
8 & 1.02 & 0.21 & 33 & 0.6 \\
9 & 1.22 & 0.36 & 52 & 2.3 \\
10 & 0.68 & 0.10 & 6.1 & $4 \mathrm{E}-05$ \\
11 & 0.96 & 0.18 & 28 & $2 \mathrm{E}-03$ \\
12 & 1.29 & 0.53 & 60 & 0.8 \\
13 & 0.62 & 0.08 & 3.4 & $1 \mathrm{E}-11$ \\
14 & 0.95 & 0.21 & 27 & $1 \mathrm{E}-04$ \\
15 & 1.27 & 0.59 & 58 & 0.3 \\
CDM & 1.33 & 0.47 & 62 & 30 \\
CHDM & 0.86 & 0.16 & 18 & $1 \mathrm{E}-02$
\end{tabular}


Table 2: continued

\begin{tabular}{ccccc}
\hline \hline Model & $\sigma_{\mathbf{8}}$ & $\boldsymbol{\Gamma}$ & $N(>M)$ & $\boldsymbol{\Omega}_{\mathrm{g}}$ \\
\hline Observ. & $0.90 \pm 0.05$ & $0.25 \pm 0.05$ & $(4-6)$ & $2.2 \pm 0.5$ \\
& & & & \\
& & $\Omega_{b}=0.08$ & & \\
16 & 1.03 & 0.28 & 34 & 6.0 \\
17 & 1.20 & 0.31 & 50 & 8.8 \\
18 & 1.26 & 0.33 & 55 & 8.9 \\
19 & 0.81 & 0.16 & 14 & 0.4 \\
20 & 1.05 & 0.21 & 36 & 1.9 \\
21 & 1.26 & 0.30 & 55 & 4.3 \\
22 & 0.74 & 0.10 & 9.1 & $3 \mathrm{E}-03$ \\
23 & 1.01 & 0.18 & 32 & 0.2 \\
24 & 1.21 & 0.35 & 51 & 1.8 \\
25 & 0.64 & 0.08 & 3.9 & $2 \mathrm{E}-07$ \\
26 & 0.95 & 0.17 & 27 & $4 \mathrm{E}-03$ \\
27 & 1.27 & 0.48 & 59 & 0.8 \\
28 & 0.60 & 0.07 & 2.5 & $2 \mathrm{E}-15$ \\
29 & 0.93 & 0.14 & 24 & $2 \mathrm{E}-06$ \\
30 & 1.19 & 0.53 & 51 & 0.1 \\
CDM & 1.28 & 0.44 & 58 & 20 \\
CHDM & 0.82 & 0.15 & 15 & $6 \mathrm{E}-03$ \\
\hline
\end{tabular}



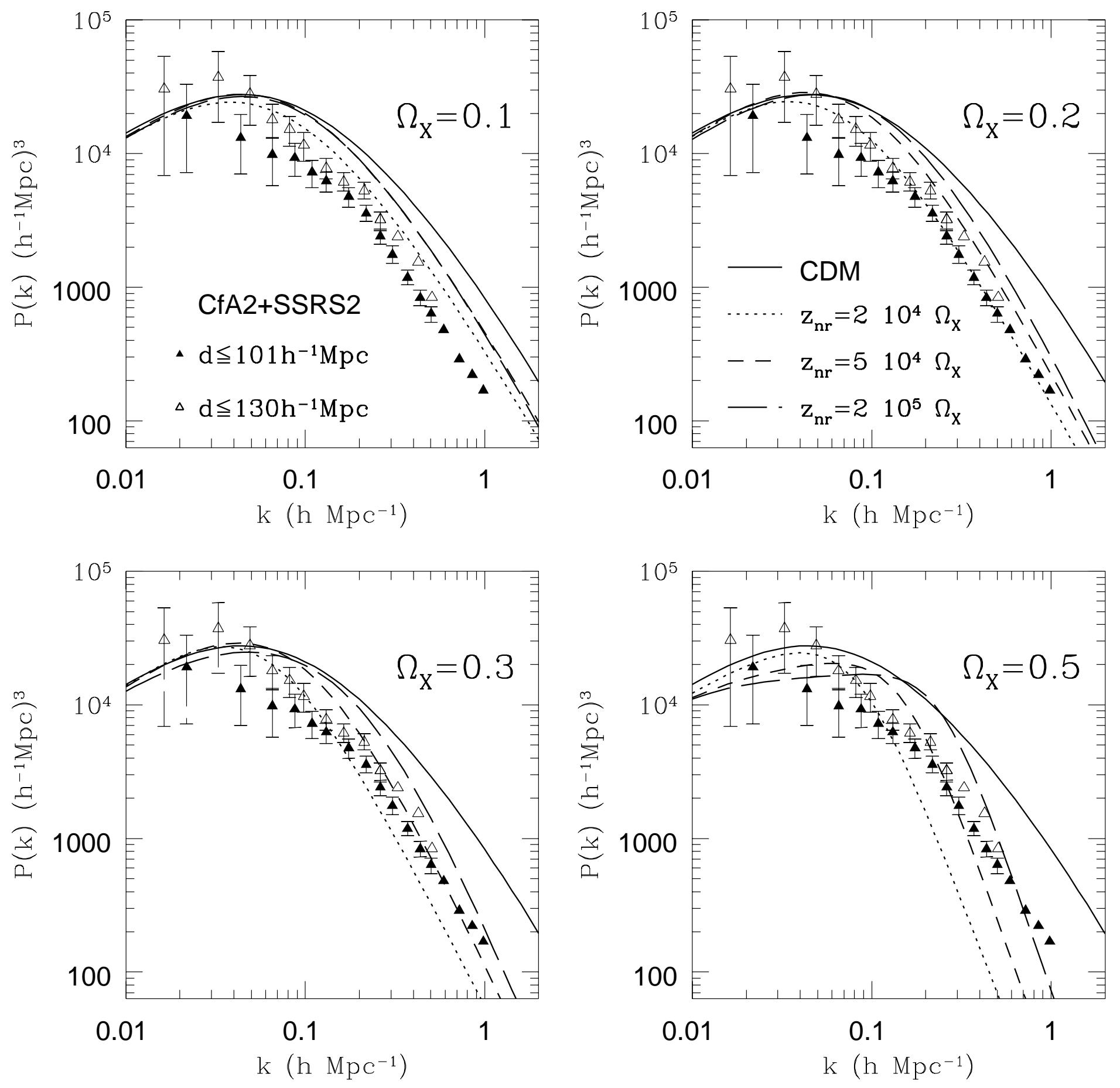

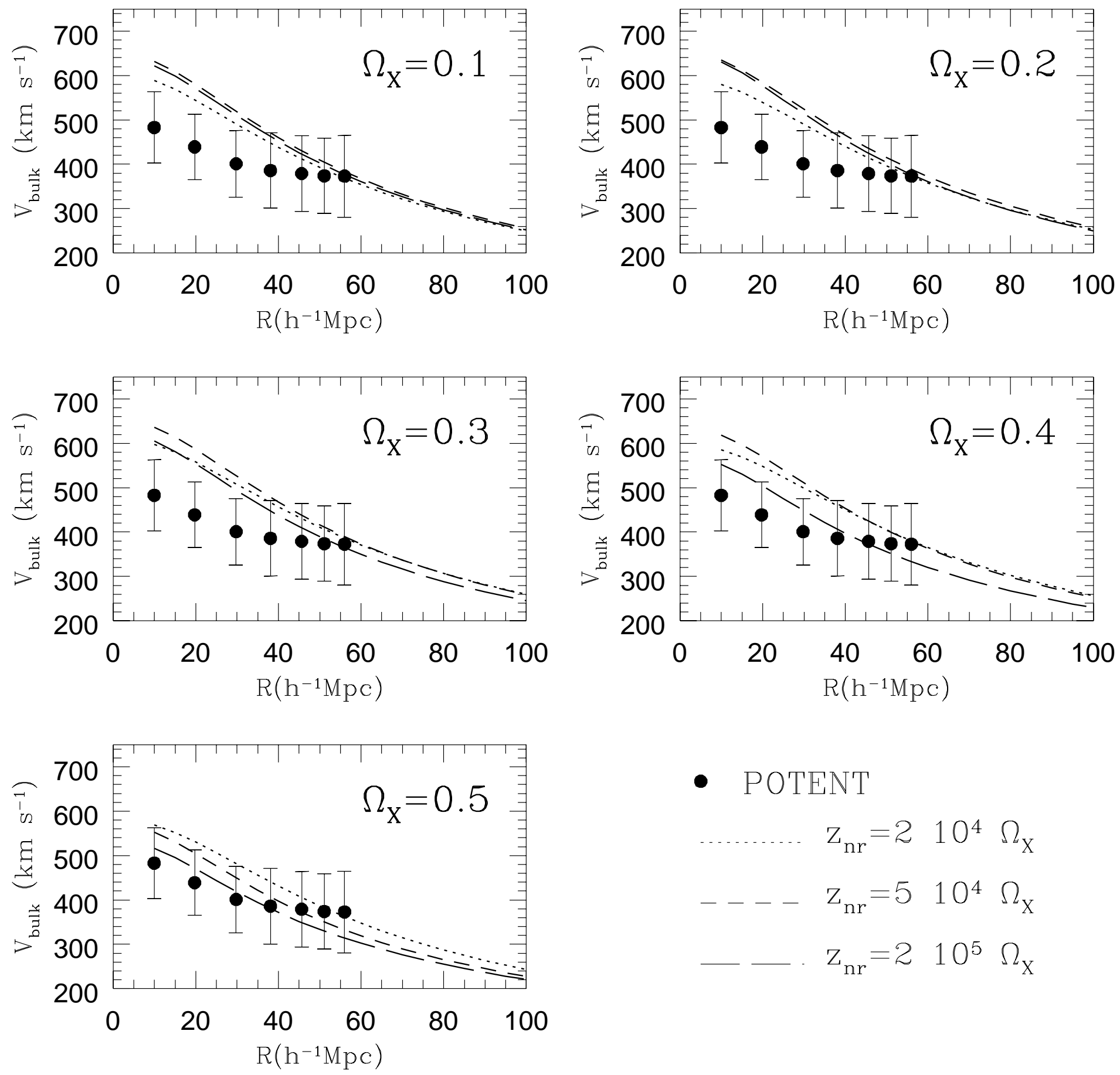

- POTENT

$$
\begin{aligned}
& \mathrm{z}_{\mathrm{nr}}=210^{4} \Omega_{\mathrm{x}} \\
& \mathrm{z}_{\mathrm{nr}}=5 \quad 10^{4} \Omega_{\mathrm{X}} \\
& \mathrm{z}_{\mathrm{nr}}=210^{5} \Omega_{\mathrm{x}}
\end{aligned}
$$



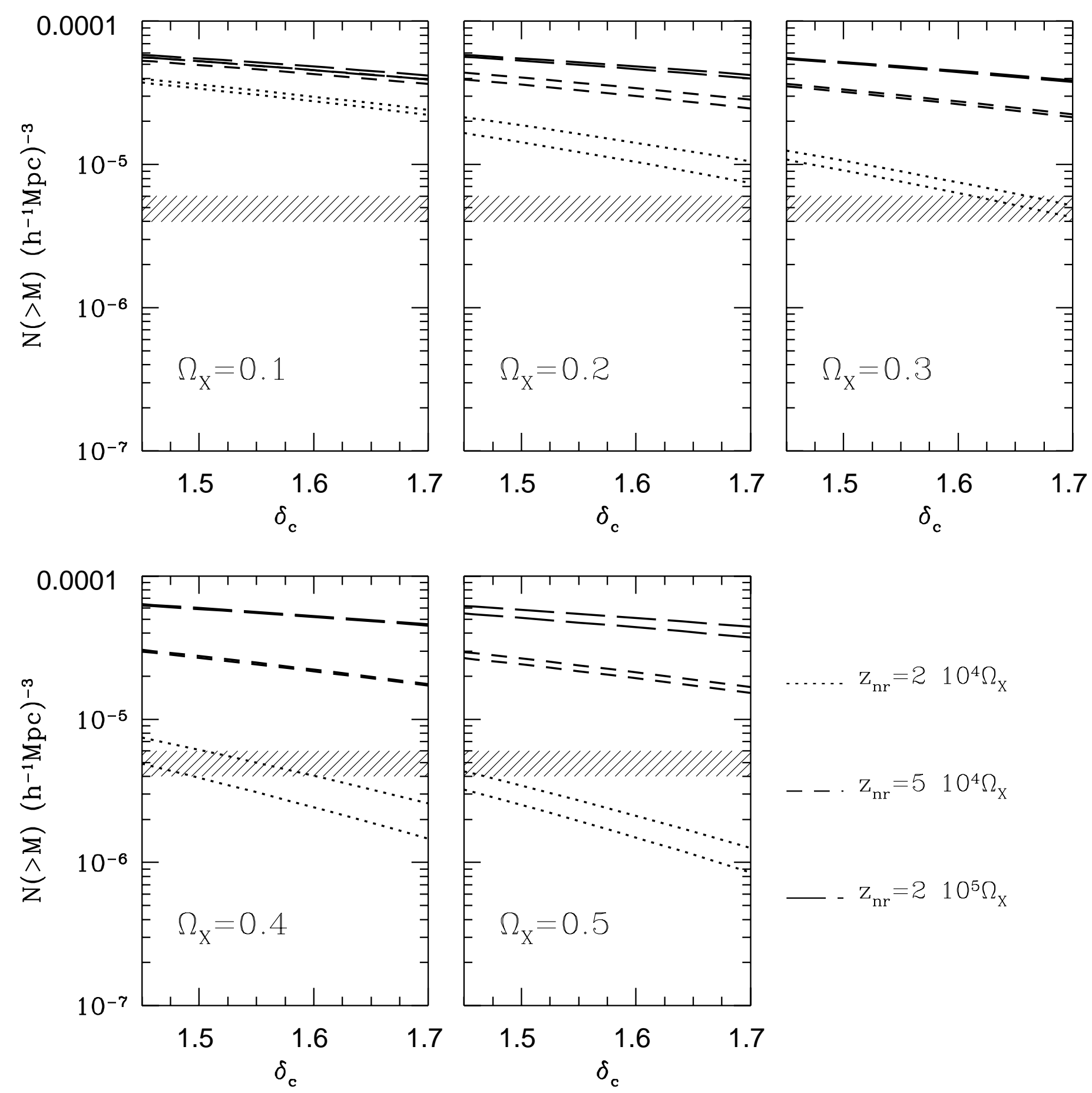

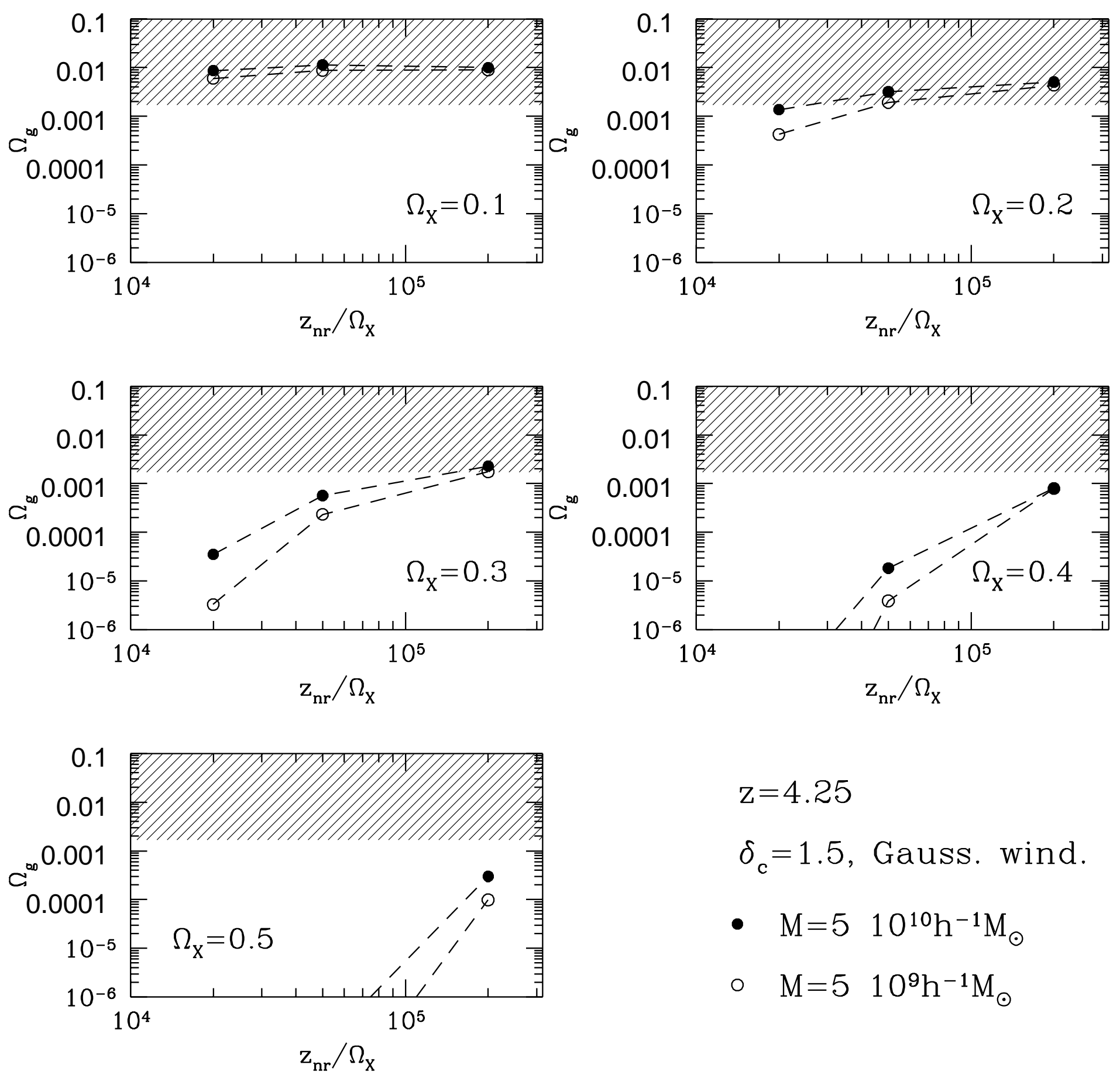

$$
\begin{aligned}
& \mathrm{z}=4.25 \\
& \delta_{\mathrm{c}}=1.5, \text { Gauss. wind }
\end{aligned}
$$$$
\text { - } \mathrm{M}=5 \quad 10^{10} \mathrm{~h}^{-1} \mathrm{M}_{\odot}
$$$$
\text { ○ } \mathrm{M}=5 \quad 10^{9} \mathrm{~h}^{-1} \mathrm{M}_{\odot}
$$ 